\section{EMIGRACIÓN, CIUDADANÍA EXTERNA Y CIUDADANÍA EUROPEA. RETOS CONCEPTUALES Y PRÁCTICOS*}

\author{
Irina Ciornei \\ Universidad Autónoma de Barcelona
}

\section{IMMIGRATION, EXTERNAL CITIZENSHIP AND EUROPEAN CITIZENSHIP. A CONCEPTUAL AND PRACTICAL APPROACH}

ABSTRACT: This paper presents a contextual analysis of the external citizenship theories and discusses their conceptual and practical linkages with the European citizenship conception. The article argues that in spite of not dwelling on the national territory, the first generation migrants may be considered legitimate members of the demos. They should, in consequence, possess rights of political participation and representation in their homeland politics. In the specific case of the European citizens, the external voting rights are a resource not only against the country of origin institutions, but also in relation to the country of residence administrations.

KEY WORDS: Immigration; external citizenship; external voting rights; representation; European citizenship.

\section{INTRODUCCIÓN}

La ciudadanía externa ${ }^{1}$ es una práctica política generalizada hoy en día. La mayoría de los estados del mundo reconocen, en mayor o menor medida, una serie de derechos de los cuales pueden gozar sus ciudadanos que viven fuera de las fronteras nacionales. El derecho central es el de poder mantener la nacionalidad, independientemente del estatus residencial (Bauböck 2007, Bauböck y Guiraudon 2009; Gamlen 2008). Este derecho básico que preserva la pertenencia en la comunidad política de origen sin tomar en cuenta la dimensión territorial, permite tener derechos en otras esferas. Por ejemplo, contiene el derecho a protección diplomática, al retorno y a la transmisión inter-generacional de la nacionalidad. En muchos casos, los ciudadanos no-residentes pueden participar y ser representados en las estructuras políticas de su país de origen.

Varios autores consideran que el concepto de ciudadanía en el exterior implica un giro en la forma de entender la ciudadanía, anteriormente asociada con la presencia en un territorio (Velasco 2009, Bauböck 2007, Bauböck y Guirau-
RESUMEN: Este artículo presenta un análisis contextual de las teorias sobre la ciudadanía en el exterior, asi como sus relaciones conceptuales y prácticos con la ciudadanía europea. Aunque la residencia en otro país puede ser un factor que cuestiona el derecho de participación y representación política de los no-residentes, consideramos que los emigrantes de primera generación son miembros legítimos del demos. En consecuencia, deberian tener derechos de participación y representación en su país de origen. Sin embargo, en el caso concreto de los ciudadanos europeos, los derechos políticos en el exterior constituyen un recurso no sólo en relación a los gobiernos de origen, sino también frente a las administraciones de los paises de residencia.

PALABRAS CLAVE: Inmigración; ciudadanía externa; derechos de voto en el exterior; representación política; ciudadanía europea.

don 2009). La ciudadanía en el exterior cuestiona, tanto en la teoría como en la práctica, las fronteras nacionales y la congruencia entre territorio-ciudadanía-democracia (Spiro 2006). Los avances tecnológicos que permiten la conexión (virtual) entre el individuo y su país de origen y el conjunto de políticas que los estados desarrollan para mantener vínculos transnacionales con sus comunidades de emigrantes desafían los principios de la política territorial. En dicha concepción, las fronteras nacionales están percibidas como los márgenes que delimitan la relación entre el ciudadano y su gobierno, y el punto donde empieza el imperio de los demás estados soberanos (Bauböck y Guiraudon 2009).

Más allá de los desafíos conceptuales, uno de los atributos de la ciudadanía externa que más polémica ha generado, tanto en los círculos académicos como en el mundo de la política, se refiere al voto desde el exterior. El derecho a voto es un atributo esencial de la ciudadanía y de la relación entre representantes y representados y por esta razón está estrechamente ligado a la residencia en el territorio de una comunidad política. (Bauböck 2007; Eva 
Østergaard-Nielsen 2003). Los mexicanos en el exterior han tenido más de cinco décadas de lucha y debates para obtener el derecho a voto en las elecciones generales de su país de origen (Smith 2008). Los italianos que viven en el exterior han visto su derecho reconocido sólo después del año 2000, aunque sus demandas de participación en las elecciones italianas llevaban ya más de 30 años. Los principales partidos británicos han debatido intensamente en los años 80 si los expatriates deberían poder votar en las elecciones generales o no, reconociendo el derecho a participación, pero con varias limitaciones. En otros casos, el proceso del reconocimiento del voto desde el exterior ha sido menos contencioso. Los españoles en el exterior gozan de igualdad de derechos con sus co-ciudadanos residentes en la constitución de 1978. Los rumanos y los polacos viviendo en el extranjero también vieron su derecho reconocido después de la caída del régimen comunista. Francia y EEUU son países pioneros en reconocer los derechos de participación política de sus ciudadanos residentes fuera de las fronteras nacionales, empezando en la década de los 70 .

En las últimas décadas, se puede observar una tendencia creciente entre los estados de otorgar derechos de participación desde el exterior (Rhodes y Harutytunyan 2010). Con pocas excepciones, la mayoria de los países del mundo han extendido el sufragio hacia el exterior después de la década de los 70 y han facilitado el acceso a voto a través de reformas institucionales, creando mecanismos como el voto electrónico o el voto por correo. Según Ellis et al. (2007) 115 de 214 países reconocen el derecho de participación política de los ciudadanos que viven en el exterior. En una investigación más reciente, Collyer (2011) encontró que 129 estados reconocen derechos políticos a los ciudadanos no residentes. La mayoría de estos estados (93) permiten el voto desde el exterior (consulado, correo y/o electrónico) para representantes en una circunscripción doméstica. En un número más reducido de casos (23) los emigrantes tienen que volver a su país el día de las elecciones y votar en la circunscripción que les corresponde. Algunos países (13) reconocen una circunscripción separada en la que los emigrantes pueden elegir sus propios representantes en el parlamento.

La ciudadanía europea es otro ejemplo de institución que desafía la dimensión nacional-territorial de la ciudadanía moderna. Desde hace décadas, los europeos se pueden mover y trabajar libremente en otros estados miembros en igualdad de condiciones con los ciudadanos nacionales². Esta "ciudadanía de mercado" cómo ha sido llamada por varios autores (Everson 1995; Bellamy y Warleigh 2001) ha sido progresivamente complementada por una dimensión política, que presupone derechos de participación y representación a nivel local en los lugares de residencia y derechos de voto para el Parlamento Europeo. A pesar del carácter inovador de la ciudadanía europea en relación a nuevas formas de participación y representación más allá de la nacionalidad y la territorialidad, hay un derecho central que los arquitectos de la construcción europea han dejado al lado: derechos políticos en las elecciones nacionales de los países de residencia de los migrantes intra-europeos. Shaw (2007) considera que desvincular la nacionalidad de la plena participación política es un atributo de la soberanía que los estados miembros no están dispuestos a negociar.

La relación entre los migrantes intra-europeos y sus estados de origen ha sido poco contemplada en el contexto de la ciudadanía europea. No obstante, según el derecho comunitario, tener la nacionalidad de un estado miembro es la condición necesaria para que un ciudadano disfrute de los derechos de la ciudadanía supranacional en otro estado miembro. Así mismo, para las elecciones europeas, un ciudadano que reside en otro país europeo puede optar a votar para candidatos de su estado de nacionalidad o para los de su país de residencia.

A pesar de la expansión del voto de los ciudadanos no residentes en la mayoría de los países del mundo y de la multiplicación de los debates académicos sobre el tema, solamente en el último informe de la Comisión sobre la ciudadanía europea (Comisión Europea 2010) se señala el hecho de que varios estados miembros impiden o dificultan el ejercicio del voto para sus ciudadanos que disfrutan de sus derechos de libre circulación. La comisión invita al diálogo a sus miembros para reflexionar sobre cómo compaginar los derechos de la ciudadanía europea con la participación política en el país de origen.

Como apuntábamos al principio del texto, la participación política desde el exterior es una tendencia en expansión de las políticas de ciudadanía de los estados. No obstante, varios autores consideran que su justificación teórica no es tan sencilla (López-Guerra 2005; Bauböck 2007, 2009; 
Owen 2011). ¿Cómo argumentar que individuos que ya no están sujetos al gobierno de un país todavía pueden tener capacidad de decisión y voto en sus estructuras? Y si los ciudadanos no-residentes tienen derechos de participación en su país de origen, ¿cómo representar sus intereses? ¿Cómo acomodar los derechos de la ciudadanía europea con los derechos de participación y representación en el país de origen? ¿Son los europeos una categoría especial de ciudadanos en el exterior? La desvinculación entre ciudadanía y territorio desafía el reto para establecer nuevas formas de participación y representación extraterritoriales.

En los siguientes apartados vamos a abordar esas tres cuestiones relacionadas con la ciudadanía en el exterior -la participación y representación política externas y su acomodación con los principios de la ciudadanía europeadesde la perspectiva de la teoría política contextual ${ }^{3}$. El artículo tiene principalmente un enfoque analítico, pero usa también estudios empíricos y fragmentos de trabajos de campo que se han hecho previamente con las comunidades rumanas en España ${ }^{4}$.

Partiendo de autores como Bauböck (2007), argumento que los derechos de participación y representación de los ciudadanos no-residentes son justificables dentro de la teoría política liberal. La participación política desde el exterior no es un derecho universal que se transmite intergeneracional entre los nacionales no-residentes. Tampoco es una práctica política que no se puede legitimar dentro de la teoría normativa liberal. La participación política externa es un derecho que depende de las circunstancias vitales y los "lazos genuinos" de cada emigrante con su pais de origen.

Responder en afirmativo a la pregunta si ciertas categorías de ciudadanos no-residentes forman parte del demos nos lleva a la cuestión relacionada con su representación política. En esta perspectiva, el argumento del artículo es que a pesar de la diversidad de las comunidades residentes en el exterior, los ciudadanos externos tienen necesidades e intereses distintos en comparación con los ciudadanos residentes y por esta razón se deberían articular a través de la lógica de la representación especial (Spiro 2006).

En el caso concreto de los ciudadanos europeos, su relación política con el país de origen no está relacionada solamen- te con sus circunstancias vitales, sino también con poder ejercer los derechos asociados a la ciudadanía europea. Un europeo puede moverse libremente en el territorio de la Unión si posee el pasaporte de un estado miembro. En este caso, la puerta de entrada en los países de la UE depende de la nacionalidad de origen y no del estado miembro de residencia. Por esta razón, ejercer el derecho a libre movimiento y poder participar en la vida política del país de origen no debería ser un juego de suma cero.

Partiendo de la cuestión de la representación directa de los ciudadanos no-residentes, el artículo aporta una reflexión sobre los derechos de representación de los migrantes europeos en sus países de origen y sus posibles ramificaciones prácticas. Los derechos de representación especial de los ciudadanos no residentes constituyen un conjunto de recursos que refuerzan su nivel de integración no sólo en relación a los gobiernos de origen, sino también en los lugares de residencia. En el caso concreto de los ciudadanos europeos, la ciudadanía en el exterior y la representación directa de éstos en el país de origen pueden complementar la ausencia de la plena integración política en los lugares de residencia.

La primera parte presenta un análisis contextual de las políticas de la ciudadanía externa y apunta las principales modificaciones que ellas aportan al concepto de la ciudadanía nacional. En el segundo apartado se discuten los principales debates teóricos relacionados con la participación política en el exterior y las relaciones entre la ciudadanía externa y la ciudadanía supranacional. La tercera parte se centra en el concepto de representación de los ciudadanos en el exterior con un enfoque especial en el caso de los ciudadanos europeos.

\section{EMIGRACIÓN Y CIUDADANÍA}

La ciudadanía externa desafía la tradición de pensamiento sobre la ciudadanía nacional y la participación democrática. Ella presupone la desvinculación de la política territorial de la pertenencia y de la vita activa del ciudadano dentro de la comunidad política. La ciudadanía nacional, en términos de Marshall (1950/2009), expresa la igualdad de los que son miembros plenos de la comunidad política. La igualdad de los miembros de la comunidad (es decir, de los ciuda- 
danos nacionales) se ha logrado a través del tiempo con la expansión y el reconocimiento de los derechos civiles, políticos y sociales. La definición de Marshall supone una clara delimitación entre miembros-ciudadanos/extranjeros y entre territorio nacional/exterior. En consecuencia, un elemento central de la concepción nacional de la ciudadanía es la residencia/presencia en el territorio. Aunque la movilidad transfronteriza y la migración son prácticas anteriores a la formación de los estados nacionales, la teoría de la ciudadanía nacional ha dado por supuesto la dimensión territorial tanto en relación a la identidad del individuo como en relación a su participación política.

Barry (2006) define la ciudadanía en el exterior como el conjunto de políticas diseñadas por los estados para mantener los vínculos con los ciudadanos que residen temporalmente o permanente fuera de las fronteras nacionales y el conjunto de prácticas (económicas, culturales y políticas) que los emigrantes desarrollan en relación a su país de origen. Los vínculos entre el ciudadano y su estado ya no se construyen en torno a la idea de sujeción a las leyes que el mismo demos diseña para sí (Dahl 1989; LópezGuerra 2005). Fuera de las fronteras nacionales, el elemento clave que permite tener derechos en la comunidad de origen es la identidad nacional. Por esta razón, Joppke (2003) considera que la ciudadanía externa presupone la re-etnicización del concepto liberal de ciudadanía.

Las principales medidas a través de las cuales los estados mantienen los vínculos con sus ciudadanos en el exterior se extienden en varios ámbitos: políticas económicas para atraer y canalizar las remesas; políticas culturales a través de las cuales se recrea la identidad nacional fuera del pais y se subvenciona el aprendizaje de la lengua de origen; políticas sociales a través de las cuales se facilita el retorno, el acceso temporal o permanente a ciertos servicios en el país de origen o el acceso a sistemas de ayudas; reformas burocráticas y electorales para diseñar espacios de participación y representación para los ciudadanos en el exterior (Barry 2005; Levitt y La Dehesa 2003). De este conjunto de medidas, el reconocimiento del derecho a voto desde el exterior es una de las más controvertidas en la práctica y el tema más debatido en la literatura sobre la ciudadanía externa.

Spiro (2006: 209) considera que los derechos políticos son un componente central de la institución de la ciudadanía moderna puesto que marcan la distinción entre ciudadano y extranjero. Desde una perspectiva histórica, marcan la transformación del súbdito en ciudadano. Es más, en la mayoría de las democracias occidentales, los inmigrantes residentes no tienen derechos políticos sin ser naturalizados, mientras que los ciudadanos en el exterior, aún de segunda y tercera generación, pueden participar sin restricciones en las elecciones nacionales.

Las razones por las cuales los estados otorgan derechos políticos a los ciudadanos en el exterior no son necesariamente transparentes. La mayoría de los gobiernos argumentan que los emigrantes "se merecen" un estatus especial dentro del demos, ya que su salida del país fue un sacrificio para mejorar las condiciones de vida de su entorno (Østergaard-Nielsen 2003). En muchos casos, los ciudadanos en el exterior pasan de "ausentes" a héroes (véase el caso de México en Smith 2008). En otros contextos, cómo en Reino Unido por ejemplo, la integración europea y los derechos de movilidad y trabajo en los demás estados miembros han sido los argumentos principales para que los que viven fuera mantengan ciertos derechos en su país de origen. En los paises en transición, tanto en el Sur como en el Este de Europa, los derechos de la diáspora han sido reconocidos en las constituciones democráticas. En todo caso, más allá de la retórica oficial, tener un cierto control de las remesas y un lobby potente en el exterior son unas de las motivaciones principales de los estados de origen (Østergaard-Nielsen 2003; Baubock 2007). Autores como Rhodes y Harutyunyan (2010) consideran que la competición partidista en el país de origen es otro factor que explica la extensión del sufragio en el exterior.

Los lobbies pro-emigrantes argumentan que aún viviendo fuera de las fronteras nacionales, el vínculo emocional con el país de origen justifica tener derechos políticos alli. Muchos todavía tienen propiedades y familiares. Es más, con las nuevas tecnologías, la diáspora puede mantenerse informada diariamente sobre las cuestiones políticas; en otras palabras, pueden ser ciudadanos competentes y responsables, igual que los ciudadanos residentes. La mayoría de los emigrantes reclaman igualdad en derechos con los residentes nacionales. De hecho, la constitución española y el Estatuto de la Ciudadanía Española en el Exterior parte del principio de igualdad entre los ciudadanos, independientemente de su residencia. 
En la teoría liberal, el vínculo emocional con un lugar no constituye una base para otorgar derechos políticos. Tampoco la ausencia de un territorio, ya que la vida de un individuo que vive fuera está pocas veces afectada por las decisiones de su país de origen. Es más, ¿cómo puede ser la ausencia del territorio un elemento ignorado en una concepción sobre la igualdad y la justicia social y política?

\section{EMIGRACIÓN E INCLUSIÓN POLITICA}

A pesar de la práctica política favorable en relación a la participación desde el exterior, los debates teóricos se centran en dos cuestiones: primera, si los emigrantes deberian en principio tener derechos de participación en su país de origen; y segunda, si los vínculos emocionales y económicos con el país de origen son condiciones suficientes para poder participar en la vida política de allí. El primer debate aborda una pregunta más amplia, relacionada con quién tiene derecho a reclamar la ciudadanía en una comunidad política; en otras palabras, ¿cuáles son los principios según los cuales se define y se organiza un pueblo? ¿Es la pertenencia/vínculo identitario con la comunidad de origen la base suficiente para reclamar derechos de participación? El segundo debate se enfoca más bien en la práctica de la ciudadanía y se pregunta si a pesar de los vínculos emocionales y económicos, los que viven fuera pueden ser ciudadanos informados y responsables en relación a su pais de nacimiento.

\section{¿Es la participación política desde el exterior una cuestión de derechos?}

En 1999 el Consejo de Europa apuntaba en una declaración oficial que poder votar en el país de origen es una cuestión de derechos humanos. El derecho a voto es una parte esencial del proceso democrático y por esta razón los emigrantes deberían poder ejercerlo plenamente. En 2004 el mismo organismo propuso reflexionar sobre unos derechos internacionales para los expatriados que incluyan la participación política en el país de origen (Spiro 2006: 232). La pregunta que surge de estas iniciativas es ¿por qué el derecho a voto desde el exterior es un derecho humano?

Tomando como punto de partida el principio de inclusión de R. Dahl (1989), López-Guerra (2005) argumenta que los emigrantes no deberian tener el derecho a voto en su país de origen. El principio de inclusión política establece que todos aquéllos (excepto inmigrantes temporales y personas que no pueden decidir por sí mismas) que están sujetos a las leyes de un país, deberían incluirse en el demos. Es decir, deberían tener derechos de participar en la formulación de las leyes que les gobiernan. En consecuencia, la inclusión de $\mathrm{A}$ o $\mathrm{B}$ en el cuerpo ciudadano de un país no es una cuestión de derechos humanos, sino un acto que responde a un criterio de cualificación.

La definición de Arendt (1951/1973) de la ciudadanía como "el derecho a tener otros derechos" apunta a un derecho universal. Pero su contendido, tener derecho a la ciudadanía $X$, se instituye contextualmente y se guía según una serie de criterios que establecen quien puede formar parte del cuerpo de ciudadanos. Según Whelan (1983), los criterios que definen el cuerpo ciudadano no son de naturaleza democrática ya que el ejercicio de la democracia presupone un pueblo ya formado y un criterio de diferenciación entre miembros/extranjeros. Pero, presuponer que la democracia es sólo un conjunto de reglas sobre cómo tomar decisiones, significaría vaciarla de contenido (López-Guerra 2005). Dahl (1989) argumenta que debería existir un criterio de inclusión política que sea consistente con la misma idea de democracia. Su argumento se puede resumir en tres criterios: a) en una democracia, los gobiernos deben considerar por igual los intereses de los gobernados; b) cada persona es el mejor juez para su propio bien; c) en consecuencia, todos los adultos están igualmente cualificados para participar en los procesos de decisión de su comunidad política (López-Guerra 2005: 221). Por ello, según estos autores, el criterio de cualificación para formar parte del demos establece que todos aquellos gobernados por las leyes de un país deben poder participar en los procesos de toma de decisión. Resumiendo, los derechos políticos en un país se deben otorgar a los que están gobernados por las leyes del país.

Owen (2011) critica la interpretación rígida que LópezGuerra hace al principio de la sujeción democrática de Dahl. El autor considera que la presencia/residencia en un territorio no es la condición suficiente ni necesaria para que alguien esté sujeto a las leyes de un gobierno. Por ejemplo, hay leyes sobre propiedad e impuestos que afectan a todos los nacionales, independiente de su estatus residencial. Es verdad que los ciudadanos residentes están

ARBOR Vol. 188755 mayo-junio [2012] 543-556 ISSN: 0210-1963

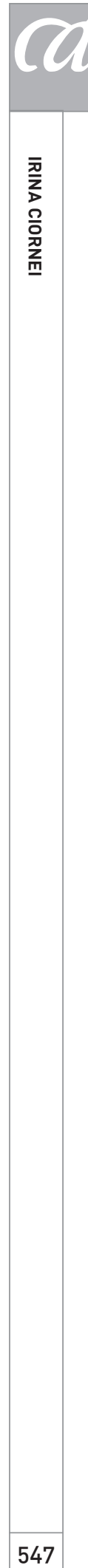


sujetos a un número más grande de leyes que los noresidentes. Pero, considera Owen, el principio de Dahl no se debe interpretar en una manera cuantitativa (ibid.: 19). El principio de la sujeción democrática no es sinónimo con el hecho que quienes están más afectados tienen derechos a hacer parte del demos. Al contrario, el principio requiere que quien está afectado, da igual en qué proporción, tiene que hacer parte del cuerpo de ciudadanos. Por esta razón el autor considera, al contrario que López-Guerra, que los emigrantes son miembros legítimos del demos.

Bauböck (2009) propone un principio alternativo de inclusión en el demos que toma en cuenta no sólo las circunstancias presentes de la vida de los individuos, sino también sus circunstancias biográficas. Según el autor, el principio de inclusión política debería referirse a todos aquéllos que tienen un interés sustancial en la comunidad política. Más especificamente, el derecho a ciudadanía en una comunidad política puede ser reclamado por: a) personas que dependen de esa comunidad para la protección de sus derechos fundamentales (criterio de la dependencia), y por b) personas que son o fueron sujetas a la autoridad de esa comunidad política durante un período determinado de su vida (criterio biográfico). Este último criterio rompe con la dimensión territorial de la ciudadanía y establece que el pasado y las circunstancias personales en su conjunto son un elemento importante que vinculan al individuo a una comunidad política. En este sentido el principio de inclusión de los stakeholders (stakeholder principle en inglés) es más amplio que el principio apuntado por Dahl, que se centra sólo en las circunstancias presentes de la vida de alguien. Aplicado al concepto de ciudadanía en el exterior, Bauböck considera que el criterio de la dependencia requiere que los emigrantes de primera y segunda generación puedan retornar a su pais, es decir, que puedan retener la nacionalidad de origen. Pero, en lo que concierne los derechos de participación política, sólo los emigrantes de primera generación cumplen con el criterio biográfico. El argumento de Bauböck justifica las prácticas políticas sobre el voto en el exterior, pero critica el hecho de que los derechos de participación política se transmitan entre generaciones más allá de la primera generación de emigrantes.

\section{Prácticas económicas, responsabilidad y ciudadanía}

Otro conjunto de debates sobre el derecho de voto desde el exterior se centra en los factores empíricos que pueden jus- tificar o al contrario, obstaculizar, la participación política de los ciudadanos no-residentes. Las prácticas económicas transnacionales han constituido una herramienta útil en el proceso de reconocimiento de derechos para la ciudadanía en el exterior. Por su parte, los emigrantes que envían remesas o siguen pagando impuestos en su pais de origen han argumentado que ésta es una base suficiente para la participación política (Østergaard-Nielsen 2003). "No taxation without representation", decian los Tories en el Parlamento británico a mediados de los ochenta a favor de los derechos a voto de los expats. A la vez, muchos estados han considerado que otorgar voz y voto a los emigrantes les facilitaría controlar y canalizar el flujo de remesas e impuestos que éstos pagan (Bauböck 2007; ØstergaardNielsen 2003). Rubio-Marín (2006) argumenta que la contribución económica de los emigrantes es más similar a la de los agentes económicos del país que a un sistema de tasación. Las remesas son una premisa importante para el crecimiento económico, pero no una virtud ciudadana.

\footnotetext{
"Expatriates' economic contribution is of a different kind in that it results from personal choices aimed at maximizing individual profit or advancing personal interests. In assessing the political relevance of this kind of contribution, it should be compared to that of entrepreneurs and successful economic agents in the country. In doing so, we come to realize that although the activities of the latter group may indeed be a great asset to the joint economic pursuit, there is no connection between this contribution and the recognition of political rights. Being economically productive is not a required civic virtue in our democracies poor artists count as much as rich entrepreneurs in terms of vote" (Rubio-Marín 2006: 133).
}

Otro tipo de virtud ciudadana que los autores han cuestionado en relación a la participación política en el exterior se refiere a la responsabilidad y la legalidad del voto. Dada la desconexión y lejanía (física) con el país de origen, es posible que el ciudadano no esté bien informado o que vote sin mucha responsabilidad. Pero, como argumenta Spiro (2006), las nuevas tecnologías permiten que los que viven en el exterior estén al día de la situación de sus respectivos países. En todo caso, aunque estén bien informados, sentirian las consecuencias de su elección mucho menos que los ciudadanos residentes. No obstante, el porcentaje de votantes en el exterior es mucho menor a los residentes y los que eligen participar lo hacen porque tienen un interés 
especial en la política de su país de origen. Por esta razón se puede considerar que son un grupo "auto-seleccionado" que vota de una manera responsable (idem: 223).

Resumiendo, el voto en el exterior es un concepto contestado tanto en la teoría como en la práctica dados los desafíos que presupone a las concepciones clásicas de ciudadanía y participación política. Según los autores, la simple contribución económica en términos de remesas no es una condición suficiente para reclamar derechos políticos. La cuestión central es determinar por qué y en qué condiciones los emigrantes son reconocidos cómo parte del cuerpo de ciudadanos que pueden tener voz y voto en las instituciones que les gobiernan. Según Bauböck (2009), los emigrantes de primera generación todavía se pueden considerar miembros plenos en sus países de origen, dados los fuertes vínculos que han tenido y que siguen teniendo alli. Una pregunta que surge en este contexto es si los ciudadanos europeos que viven en otros Estados miembros son un caso "especial" de ciudadanos en el exterior, ya que la dicotomía entre ciudadanos nacionales/extranjeros dentro de la UE no es tan clara como en el caso de terceros países.

\section{Ciudadanía europea y ciudadanía en el exterior}

Dentro del contexto europeo, unos de los factores que han determinado el reconocimiento del voto desde el exterior para los ciudadanos no-residentes han sido la migración intra-europea y la otorgación de derechos a voto para el parlamento Europeo (Lafleur 2011). El caso de Reino Unido es ilustrativo en este sentido. Aunque la emigración de Reino Unido se ha expandido desde finales del siglo XVIII, no es hasta los principios de los 1980 que las asociaciones de expats y políticos conservadores han empezado a reclamar derechos de voto en las elecciones británicas. Uno de los principales exponentes del voto de los británicos en el exterior, miembro de los Tories, argumentaba en el Parlamento británico:

"I place this matter really in the same category, in terms of national elections, as whether a Londoner should be disenfranchised if he moves to Glasgow, or a person from Manchester if he moves to Cardiff. Such a thing would be wrong, and I hope your Lordships will agree that it is equally wrong for a British subject to be disenfranchised simply because he lives and works in Paris, in Brussels or in Rome" (Lord Bethell, Partido Conservador, Debates House of Lords, 14.03.1983).
En la misma línea, tres décadas más tarde, la Comisión Europea apuntaba en su último informe sobre ciudadanía que se debería abrir el diálogo sobre el derecho a sufragio en el país de origen a los ciudadanos europeos que viven en otro Estado miembro (Comisión Europea 2010).

Los derechos políticos de los emigrantes europeos han sido poco debatidos dentro de la literatura sobre la ciudadanía externa. Una de las razones principales es que los países europeos fueron estudiados pocas veces desde la perspectiva de la emigración. No obstante, más de 12 millones de europeos han hecho uso de sus derechos de libre circulación, residiendo en otro Estado miembro (Eurostat 2009). Teniendo en cuenta la ausencia de barreras territoriales en el ámbito económico y laboral, la pregunta que surge es si el derecho a libre circulación y la configuración de la ciudadanía europea implican mantener los derechos políticos en el país de origen.

Kochenov (2009) considera que la privación de derechos a voto en el exterior de los emigrantes intra-europeos entra en contradicción con el principio de libre movimiento y la construcción del mercado común. El autor argumenta que en los casos de los países que no otorgan o que limitan el derecho a voto en el exterior ${ }^{5}$, sus ciudadanos tienen que optar entre el derecho fundamental de elegir y ser elegido y el derecho a libre movimiento. Por esta razón los derechos políticos en el exterior de los ciudadanos UE son un caso distinto de los derechos políticos en el exterior en general.

No obstante, la solución "lógica" al problema de participación política de los migrantes intra-europeos sería el reconocimiento de derechos políticos en las elecciones nacionales de los paises de residencia. Pero, como reconocen varios autores (Shaw 2007, Wiener 1998), otorgar derechos políticos en elecciones legislativas y presidenciales en los estados miembros de residencia hubiera sido un compromiso demasiado arriesgado para la pérdida de soberanía de los estados. Por esta razón, los europeos que se mueven en otros estados miembros tienen igualdad de trato con los nacionales en las elecciones municipales y europeas, pero no pueden votar en las legislativas sin estar nacionalizados. La solución al problema del sufragio de los migrantes intra-europeos parece ser más bien un compromiso que una cuestión de principio: si es poco probable que los Estados miembros vayan a extender los derechos políticos de los ciudadanos europeos en elecciones legislativas, en- 
tonces al menos hay que reconocer estos derechos en sus Estados de origen. El uso del derecho a libre movimiento no puede causar la privación del derecho central de la ciudadanía: participar en las elecciones nacionales.

David Owen (2011) construye un argumento similar, destacando que un derecho cívico como la libertad de movimiento no debería cancelar el principio de la igualdad política (idem: 5). En otras palabras, la libre circulación tampoco debería crear situaciones en las cuales hay europeos que tienen más de un voto en elecciones generales (en su país de origen y en su país de residencia). Según el autor, hay cuatro escenarios posibles para evitar que los emigrantes europeos no se queden privados de derechos políticos en general una vez que usan el derecho a la libre circulación: a) derechos políticos en el Estado miembro de residencia; b) derechos políticos en el Estado de nacionalidad; c) la elección entre (a) y (b); d) una combinación de (a) y (b) que empieza con (a) y después de un período pasa a (b). Owen considera que la mejor solución sería lo que llama $(b+)$ : derechos de voto en el Estado de nacionalidad, con la condición de que se facilite la naturalización de los europeos residentes en otros Estados miembros. En consecuencia, el autor apuesta por otorgar derechos de participación en las elecciones generales del país de origen si los emigrantes intra-europeos no tienen dicho derecho en los Estados de residencia: "Since EU citizenship is acquired through citizenship of a member state, the primary responsibility for ensuring that an EU citizen is not politically disadvantaged by exercising the right of free movement lies with the MS through whom EU citizenship is acquired and not the MS in which the EU citizenship is resident" (Owen 2011: 5).

Si bien es verdad que la libre circulación no debería presuponer la exclusión política, los argumentos de Kochenov (2009) y de Owen (2011) parten de una concepción unilateral sobre los derechos políticos. Como hemos especificado antes, la participación en elecciones no es un derecho general, sino un derecho en relación con una comunidad política en concreto. Por esta razón, si argumentamos a favor de los derechos políticos de los emigrantes intraeuropeos en su país de origen, es porque hay un principio que lo justifique. Los derechos políticos en las elecciones generales del país de origen no deberían ser vistos como el substituto de los derechos de participación en el país de acogida.
En todo caso, el pasaporte de un Estado miembro es la puerta de entrada para el disfrute de los derechos de la ciudadanía europea; aún más cuándo una importante proporción de emigrantes intra-europeos son circulares o usan sus derechos a libre circulación en más de un Estado miembro. Dada la temporalidad y movilidad de las trayectorias laborales de los europeos, la privación de derechos políticos en el Estado de origen una vez que el ciudadano cruza la frontera sería una solución extrema que no toma en cuenta esa realidad.

Tampoco es evidente la necesidad de naturalización en el Estado miembro de residencia para adquirir derechos políticos. La innovación que conlleva la ciudadanía europea es la construcción de un espacio político y una forma de pertenencia que sobrepasan las restricciones nacional-territoriales de la ciudadanía moderna. Arraigar los derechos de participación política de los inmigrantes en la naturalización sería reafirmar un concepto nacional-territorial de la ciudadanía. Por esta razón, una combinación de derechos políticos en el país de origen y de residencia ( $\sin$ la necesidad de la naturalización) es una solución que se adapta mejor a la arquitectura de la UE.

Resumiendo, los derechos políticos de los emigrantes intra-europeos en su país de origen no deberían ser vistos como la solución para corregir la falta de participación en la política nacional de los países de residencia. El voto en el exterior de los emigrantes europeos puede ser una opción para que este grupo de ciudadanos no se quede totalmente excluido de la política nacional en general. Pero no soluciona el problema de la exclusión política en los países de residencia. No obstante, hay varias configuraciones del derecho a voto en el exterior en los cuales los europeos que viven en otro Estado miembro, y los emigrantes en general, quedan más "protegidos" que en otras. Dichas configuraciones tienen que ver con los principios de representación de la ciudadanía en el exterior.

\section{EMIGRACIÓN Y REPRESENTACIÓN}

Si bien los derechos de participación en las elecciones del país de origen han sido una cuestión debatida en la literatura, el concepto de representación de los ciudadanos residentes en el exterior ha generado menos interés acadé- 
mico. No obstante, el reconocimiento de la representación especial en el país de origen es una medida que empieza a ganar popularidad entre los gobiernos. El caso rumano es un ejemplo ilustrativo para reflexionar sobre los argumentos a favor o en contra de la representación especial de la Diáspora. Durante las elecciones parlamentarias de 2008, los rumanos en el exterior pudieron votar por primera vez a los parlamentarios de la Diáspora. Según la ley electoral que se cambió pocos meses antes de las elecciones, cuatro diputados y dos senadores estaban destinados a representar, según la dispersión geográfica, a los rumanos que residen en el exterior ${ }^{6}$. La campaña electoral de Marian Popescu, candidato del Partido Social Demócrata Rumano (PSD) en la Diáspora y el presidente de PSD en España, prometía mejorar la situación de los emigrantes en dos países: a) facilitarles el retorno y negociar con el gobierno rumano para una rápida inserción laboral; b) a los que se decidieran quedar en España, mejor diálogo con las autoridades españolas y regionales y varios programas de inserción social de la comunidad gitana. Tanto Popescu como los demás representantes de la Diáspora viven entre dos países y son conocidos en la política local y regional del pais de residencia y, obviamente, tienen buena relación con sus compañeros políticos en Bucarest. Normalmente consiguen varias facilidades para su comunidad en los lugares de residencia y aunque los cambios no son visibles a nivel agregado, los rumanos que viven cerca de una filial en el exterior de un partido de su país de origen, tienen más canales de diálogo con la política local y regional en España?.

Rumania no es el único país que reconoce una circunscripción electoral en el exterior. Francia y Portugal fueron pioneras al otorgar derechos de representación especial de los emigrantes en el parlamento nacional (Ellis et al. 2007). Después de décadas de demandas por parte de las comunidades en el exterior, Italia otorgó a partir del año 2001 derechos de participación y representación a la diáspora (Battiston y Mascitelli 2008). Tras el cambio de la constitución en 2008, los franceses en el exterior gozan no sólo de representación especial en el Senado, pero también de representación en la Asamblea Nacional por 12 diputados que representan varias áreas geográficas del mundo.

Partiendo de las diferentes prácticas de representación de los ciudadanos en el exterior, Spiro (2006) distingue entre representación asimilacionista y representación discreta o directa (discrete representation). En el caso de la representación asimilacionista, los ciudadanos en el exterior votan a candidatos en una circunscripción electoral doméstica ${ }^{8}$, mientras que en el otro caso eligen sus propios representantes. El autor considera que en función del tiempo de residencia en el exterior, o de la naturaleza del proyecto migratorio, las comunidades de emigrantes pueden llegar a tener intereses distintos. Por ejemplo, los que tienen poco tiempo desde que se fueron del país de origen o los que intentan volver continúan teniendo fuertes vínculos con los lugares de origen y en consecuencia, intereses comunes con los otros votantes residentes en la circunscripción. A contrario, los emigrantes que llevan muchos años viviendo fuera de su país de origen pueden sentirse menos identificados con los intereses locales de donde partieron, pero no obstante, pueden tener fuertes vínculos con el interés en la política a nivel nacional. En esta situación, los emigrantes constituirian un grupo distinto, con intereses que difícilmente estarian representados por parlamentarios elegidos en una circunscripción doméstica. Es más, dichos representantes tendrían pocos incentivos y pocas posibilidades para establecer contactos y canales de comunicación con sus electores en el exterior. Igualmente, las posibilidades de los emigrantes de ser seleccionados para ser candidatos en una lista propuesta en una circunscripción doméstica serian mínimas, ya que tendrían poco acceso en las estructuras de poder de sus lugares de origen.

El argumento de Spiro (2006) sigue la lógica de representación de la filosofía de Montesquieu (1748/1989) y parte de la presuposición de que los representantes políticos son la voz de los intereses locales de la circunscripción que representan. En este caso, la creación de una circunscripción en el exterior sería justificada, ya que los emigrantes están en la mayoría de los casos desconectados del mundo local de donde partieron. No obstante, hay otra tradición de la representación, que tiene raíces en la filosofía de Edmund Burke (1790/2001) y que apuesta por la representación del interés general de la nación, aunque la elección de los candidatos sigue una base territorial ${ }^{9}$. En este caso, crear diputados o senadores de la diáspora entraría en contradicción con el principio general de representación ciudadana en el país de origen.

También en oposición al argumento a favor de la representación discreta, Bauböck (2009) considera que los expatriados no son un grupo homogéneo que pueden formar la 
circunscripción $n+1$ en el exterior. Si ellos son stakeholders en la comunidad política de su país de origen, entonces sus derechos y sus deberes están considerados a nivel individual, como en el caso del resto de individuos que se incluyen en el cuerpo de ciudadanos. Los emigrantes pueden votar y ser representados en su país de origen porque todavía tienen un interés vital allí, y no porque constituyen un grupo distinto: "We should see the expatriates not as a group united by their origins, but as individuals with different biographical ties to a country of origin. What most of them share is an interest in retaining external citizenship, but they certainly do not share the same stake in the ongoing political process that is oriented through democratic elections" (Bauböck 2009: 491).

A pesar de la diversidad socio-económica y residencial de los emigrantes, la circunscripción en el exterior se debería considerar como un intento de corregir ciertas prácticas de exclusión política de este grupo. Si su voz se ve diluida en el conjunto de los electores domésticos y si sus representantes tienen pocos incentivos para acercarse, entonces la representación discreta sería la solución para hacer presente sus intereses y sus demandas. En este caso, la dimensión territorial juega más bien el papel de identificación y politización de un grupo, igual que en otros casos la raza, el género o el origen étnico pueden constituir atributos para legitimar la representación especial (Phillips 1995; Mansbidge 2003; Kymlicka 1989).

Un argumento que critica la representación discreta de las comunidades en el exterior reclama su valor simbólico y la desigualdad en comparación con la representación de los ciudadanos residentes ${ }^{10}$. En la mayoría de los casos, alrededor de diez diputados y senadores representan a millones de ciudadanos que viven fuera de las fronteras nacionales. La proporción entre representantes/representados es mucho mayor en el caso de las circunscripciones domésticas. Pero, aunque la norma de representación que se aplica dentro y fuera de las fronteras nacionales no es equitativa sino más bien simbólica, es importante apuntar el hecho de que la representación discreta hace reflejar voces e intereses que en otro contexto no tendrian un canal para ser escuchados.

En la práctica, la circunscripción en el exterior toma en cuenta la dispersión geográfica de los emigrantes y en general asigna diputados y senadores en función de los lugares de residencia. En este sentido, en Rumania hay un diputado para Europa, otro para América y Australia, otro para Asia y otro para África. Los casos italianos y francés siguen la misma lógica de división de los electores en el exterior, aunque el número de representantes es relativamente más elevado (diez diputados en el caso italiano y once en el caso francés). Por esta razón, es muy probable que la agrupación de emigrantes por sus lugares de residencia para seleccionar los representantes refleje una cierta coincidencia de intereses y problemas comunes. A pesar de la heterogeneidad interna del grupo, los emigrantes que residen en la UE, por ejemplo, pueden tener demandas e intereses distintos de los que residen en América Latina.

En consecuencia, elegir sus propios representantes en función del país/zona de residencia es una solución para hacer escuchar voces que en otras circunstancias serian silenciadas por la multitud de los votantes domésticos. Una curiosidad que surge en este contexto es analizar la relación entre las formas de representación en el país de origen y la inclusión y participación en los lugares de residencia de los emigrantes intra-europeos.

\section{Ciudadanía europea y formas de representación en el país de origen}

En los apartados anteriores hemos argumentado que la representación directa de los ciudadanos no-residentes en su país de origen es una forma legítima para articular y promover sus intereses. La función de los representantes (Senadores o Diputados de la Diáspora) sería de escuchar y avanzar en la arena política del país de origen cuestiones relacionadas con la vida cultural, social y política de las comunidades en el exterior. Pero, como hemos apuntado en el ejemplo del candidato PSD Diáspora a las elecciones legislativas rumanas, su programa político no se refería solamente a lo que iba a hacer en relación al gobierno rumano, sino también a estrategias y acciones que iba a emprender en relación a la arena política española.

El "doble mandato" que puede tener un representante de los ciudadanos en el exterior en el contexto de residencia y en el país de origen no es una estrategia posible en cualquier circunstancia. En países como, por ejemplo, México, "tener a un extranjero que se mezcla con los asuntos políticos del país está muy mal visto"11. No obstante, en el contexto europeo el juego del doble manda- 
to del representante de la Diáspora sería no solamente posible, sino de alguna forma justificable. En un estado miembro, los representantes de las comunidades en el exterior no actuarian simplemente como representantes "extranjeros" de una comunidad de inmigrantes; ellos son al mismo tiempo miembros de partidos políticos que tienen una representación supranacional. El Parlamento Europeo juega en este sentido un papel fundamental tanto en la teoría como en la práctica de los vínculos transnacionales que surgen entre los partidos de varios países europeos.

Por ejemplo, los partidos rumanos y españoles de la misma familia ideológica han cerrado acuerdos de colaboración que se materializan durante las elecciones de los dos países. Varios representantes de la comunidad rumana en España me explicaban que durante las elecciones rumanas los partidos españoles con los cuales tienen acuerdo les facilita infraestructura para la campaña electoral (sedes para reuniones, uso de las tecnologías, etc.). De la misma forma, los militantes de los partidos rumanos con sede en España apoyan al partido hermano durante las elecciones locales españolas. En este contexto de cooperación entre partidos, los parlamentarios de la Diáspora rumana están percibidos como representantes legítimos de dicha comunidad inmigrada en España y como miembros de una familia más larga de europeos que tienen representación en Bruselas.

Intentando teorizar los hechos narrados antes, se puede pensar que la representación directa de las comunidades de emigrantes constituye un recurso más que ellos pueden activar para promover sus intereses en el contexto de residencia. Por esta razón, tener representación especial no significa sólo que los emigrantes hacen que su voz sea escuchada frente a los gobiernos de su país de origen, sino también delante de las autoridades del país de residencia. Los representantes de la Diáspora pueden jugar el papel de lobby o portavoces de la comunidad frente a niveles de poder donde los europeos no tienen derechos políticos: el nivel regional y nacional del estado de residencia.

\section{Conclusiones}

La participación y representación política de los ciudadanos no residentes ha constituido un constante objeto de debates académicos y políticos, a pesar de la expansión del voto en el exterior a partir de la década de los setenta. A nivel conceptual, la ciudadanía en el exterior y la participación política en el país de origen presuponen el reto de repensar la concepción nacional-territorial de la ciudadanía moderna. No obstante, tal como apunta Bauböck (2007; 2009), no todos los ciudadanos en el exterior deberian tener derechos políticos en su país de origen. Solamente los emigrantes de primera generación pueden ser considerados stakeholders allí. En el caso concreto de los ciudadanos europeos, hemos argumentado que aunque los derechos políticos en el país de origen no deben ser vistos como el sustituto de la participación en el estado de residencia, ellos constituyen una práctica legítima; aún más si tomamos en cuenta la circularidad y la temporalidad de los procesos migratorios intra-europeos.

La representación directa de los ciudadanos en el exterior constituye una opción a través de la cual los no residentes pueden expresar y canalizar sus demandas e intereses frente al gobierno de origen. Al mismo tiempo, puede constituir un recurso extra que ellos tienen para negociar su posición en los lugares de residencia, especialmente en el caso de la migración intra-europea. Los vínculos transnacionales entre distintas instituciones y partidos políticos y el espacio común de encuentro que es el parlamento europeo constituyen unas condiciones favorables para que los representantes del país de origen de los emigrantes tengan más capacidad de lobby y diálogo en el contexto de residencia. 
* El presente trabajo se ha realizado en el marco del proyecto I+D "Emigración, Ciudadanía externa y partidos políticos en España, Italia, Francia y Rumania" (CSO201016535, 2011-2013), financiado por el Plan Nacional I+D+i de MICINN y cuya Investigadora Principal es Eva Østergaard-Nielsen.

1 A lo largo de este artículo utilizo los conceptos "ciudadanía externa" y "ciudadanía en el exterior" como sinónimos. Los dos conceptos son la traducción de la noción de "external citizenship" que generó una serie de debates en el ámbito académico anglo-sajón en la primera década del siglo XXI. En la legislación española se utiliza el término de "ciudadanía en el exterior" a partir del año 2006, cuando se promulga el "Estatuto de la Ciudadanía Española en el Exterior".

2 El tratado de Maastricht (1992) establece los derechos relacionados a la ciudadanía europea y el principio de non-discriminación a base de nacionalidad entre los migrantes intraeuropeos y los ciudadanos residentes de los estados miembros en los ámbitos socio-económicos. No obstante, las últimas dos etapas de integración europea $(2004,2007)$ han sido acompañadas por medidas temporales que imponen restricciones para los "nuevos Europeos" en la mayoria de los mercados laborales de la UE15. Dichas restricciones tienen una duración máxima de siete años.

3 Según Carens (2004) el enfoque contextual presupone un análisis conjunto de las teorías y los estudios de casos y el diálogo constante entre el pensamiento teórico y las circunstancias empíricas a las cuales dicho pensamiento se refiere. "To summarize, the idea is to engage in an ongoing dialectic that involves mutual challenging of theory by practice and of practice by theory" (Carens 2004: 120).

4 El estudio de caso de las comunidades rumanas hace parte de una investigación doctoral y se ha realizado entre 2007 y 2010 . Se compone de entrevistas cualitativas a líderes de asociaciones y organizaciones del colectivo y a políticos locales y regionales españoles en la Comunidad de Madrid y la Comunidad Valenciana. Las entrevistas han sido complementadas con fuentes secundarias. El tema general de estudio de las dos comunidades se refiere a la incorporación política en España y a las prácticas de ciudadanía que los colectivos rumano y británico tienen en el país de origen y a nivel supranacional.

5 Los paises que no reconocen derecho a voto de los nacionales no residentes automáticamente o después de un período que estos viven fuera son: Irlanda, Hungría, Malta, Dinamarca, Reino Unido y Austria (Comisión Europea 2010).

6 Los cuatro diputados representan cuatro circunscripciones denominadas: América y Australia, Asia, Europa y África; los dos senadores representan Asia-Europa y América-Australia-África.

7 Las referencias a la comunidad rumana se basan en el estudio de caso de dicha comunidad en la Comunidad de Madrid y la Comunidad Valenciana entre 2007-2010. El estudio recoge entrevistas cualitativas con líderes de asociaciones rumanas, miembros de partidos políticos rumanos y politicos de los gobiernos locales en dichas comunidades.

8 Normalmente esta circunscripción es la capital o la circunscripción que corresponde al último lugar de residencia.
Recibido: 21 de octubre de 2011 Aceptado: 12 de noviembre de 2011 
9 Para un análisis comprehensivo del concepto de circunscripción, véase Rehfield (2005).

10 Después del cambio de la ley electoral en 2008, la mayoría de los periódicos rumanos y las asociaciones de inmigrantes decretaban que "el voto de la diáspora ha sido suprimido".

11 Conferencia de Marco Antonio Baños, Consejero Electoral del IFE México sobre el voto de los mexicanos en el exterior. Barcelona CIDOB, 07.11.2011.

\section{BIBLIOGRAFÍA}

Arendt, H. (1951/1973): The Origins of Totalitarism, San Diego, Harcourt Brace Jovanovich.

Battiston, S. y B. Mascitelli (2008): "The challenges to democracy and citizenship surrounding the vote to Italians overseas", Modern Italy, 13(3): 261280.

Barry, K. (2006): "Home and Away: The Construction of Citizenship in an Emigration Context", New York University Law Review, 81: 11-59.

Bauböck, R. y V. Guiraudon (2009): "Introduction: Realignments of Citizenship: Reassessing Rights in the Age of Plural Memberships and Multi-level Governance", Citizenship Studies, 13(5): 439-450.

Bauböck, R. (2009): "The rights and duties of external citizenship", Citizenship Studies, 13(5): 475-499.

Bauböck, R. (2006): "Stakeholder Citizenship and Transnational Political Participation: A Normative Evaluation of External Voting", Fordham Law Review, 75: 2393-2447.

Bellamy, R. y A. Warleigh (2001, eds.): Citizenship and Governance in the European Union, Londres, Continuum International Publishing Group.
Burke, E. (1790/2001): Reflections on the Revolution in France, Stanford, Stanford U.P.

Carens, J. H. (2004): "A Contextual Approach to Political Theory", Ethical Theory and Moral Practice, 7(2): 117-132.

Collyer, M. (2011): A Geography of Extraterritorial Citizenship: Explanations of External Voting. Paper presentado en la conferencia "Migrants and External Voting in the EU: New Prospects and Challenges for Research and Policymaking". Roma, Academia Bélgica, 2627 Mayo 2011.

Comision Europea (2010): Report from the Commission to the European Parliament, the Council and the European Economic and Social Committee under article 25 TFEU. On progress towards effective EU Citizenship 2007-2010. \{COM(2010) 603 final\} http://ec.europa.eu/justice/citizen/files/ com_2010_603_en.pdf - Fecha de consulta: 04.03.12.

Dahl, R. A. (1989): Democracy and Its Critics, Yale, Yale U.P.

Ellis, A. et al. (2007): Voting from Abroad: The International IDEA Handbook, n.d. http://www.idea.int/publications/voting_from_abroad/index.cfm. - Fecha de consulta: 04.03.12.

Everson, M. (1995): "The Legacy of the Market Citizen", en Shaw, J. (ed.) The New Legal Dynamics of European Union, Oxford, Clarendon Press, 7390.

Gamlen, A. (2008): "The Emigration State and the Modern Geopolitical Imagination", Political Geography, 27(8): 840-856.

Lafleur, J. M. (2011): "Why do states enfranchise citizens abroad? Comparative insights from Mexico, Italy and Belgium", Global Networks, 11(4): 481-501.

Joppke, C. (2003): "Citizenship Between De- and Re-Ethnicization", European
Journal of Sociology / Archives Européennes De Sociologie, 44(3): 429458.

Kochenov, D. (2009): "lus Tractum of Many Faces: European Citizenship and the Difficult Relationship Between Status and Rights", Columbia Journal of European Law, 15: 169.

Kymlicka, W. (1989): Liberalism, Community, and Culture, Oxford, Clarendon Press.

López-Guerra, C. (2005): "Should Expatriates Vote?", Journal of Political Philosophy, 13(2): 216-234.

Marshall, T. (1950/2009): "Citizenship and Social Class", en Manza, J. y M. Sauder (eds.): Inequality and Society, Nueva York, W. W. Norton and Co., pp. 148154.

Montesquieu, C. de S. (1748/1989): The Spirit of Laws, New York, Cambridge U.P.

Østergaard-Nielsen, E. (2003): International Migration and Sending Countries: Perceptions, Policies, and Transnational Relations, Londres, Palgrave Macmillan.

Owen, D. (2011): External Voting and the European Polity. Rome. Paper presentado en la conferencia "Migrants and External Voting in the EU: New Prospects and Challenges for Research and Policy-making". Roma: Academia Bélgica. 26-27 Mayo 2011.

Pennock, J. R. y J. W. Chapman (eds.) (1983): Liberal Democracy, Nueva York, New York U.P.

Phillips, A. (1995): The Politics of Presence, Oxford, Oxford U.P.

Rehfeld, A. (2005): The Concept of Constituency: Political Representation, Democratic Legitimacy and Institutional Design, Cambridge, Cambridge U.P.

Mansbridge, J. (2003): "Rethinking Representation", American Political Science Review, 97(4): 515-528. 
Rhodes, S. y A. Harutyunyan (2010): "Extending Citizenship to Emigrants: Democratic Contestation and a New Global Norm", International Political Science Review, 31(4): 470-493.

Rubio-Marin, R. (2006): "Transnational Politics and the Democratic Nation-State: Normative Challenges of Expatriate Voting and Nationality Retention of Emigrants", New York University Law Review, 81: 117-147.

Shaw, J. (2007): "E.U. Citizenship and Political Rights in an Evolving European Union", Fordham Law Review, 75: 2549-2573.
Smith, R. C. (2008): "Contradictions of Diasporic Institutionalization in Mexican Politics: The 2006 Migrant Vote and Other Forms of Inclusion and Control", Ethnic and Racial Studies, 31(4): 708-741.

Spiro, P. J. (2006): "Perfecting Political Diaspora", New York University Law Review, 81: 207-233.

Velasco, J. C. (2009): "Transnacionalismo migratorio y ciudadanía en mutación", Claves de razón práctica, 197: 32-41.

Whelan, F. G. (1983): "The Democratic Theory and the Boundary Problem", en Pennock, J. R. y J. W. Chapman (eds.): Liberal Democracy, New York, New York U.P., 13-47.

Wiener, A. (1998): European citizenship practice. Building institutions of a non-state, Boulder, Westview Press.

\section{Debates Parlamentarios/ \\ Parlamento Británico:}

http://hansard.millbanksystems.com/ lords/1983/mar/14/european-assemblyelections-amendment\#S5LV0440PO_ 19830314_HOL_180. Fecha de consulta 04.03 .12 